\title{
Traceable calibration of automatic weighing instruments operating in dynamic mode
}

\author{
Rafat Sarnecki ${ }^{1, *}$, Wojciech Wiśniewski ${ }^{1}$, Wiktor Ślusarski ${ }^{1}$ and Piotr Wiłkojć ${ }^{1}$ \\ ${ }^{1}$ Central Office of Measures, Laboratory of Mass, Elektoralna 2 Warsaw, Poland
}

\begin{abstract}
This article describes calibration method of automatic weighing instruments for measuring vehicles in motion. These measuring instruments in future will be widely used to determine the axle loads, if applicable the axle-group and vehicle mass of road vehicles when the vehicles are weighed in motion. For several years, some activities have been carried out on the weight control system that determines the mass of vehicles moving at higher speeds, called HS-WIM. The implementation of such systems will affect both the improvement of road safety and indirectly limit the number of road users moving by overloaded vehicles.
\end{abstract}

\section{Introduction}

Automatic instruments for weighing vehicles in motion (WIM) [1], are one or two-bridging measuring instruments used to determine the axle load, axle group load and total vehicle mass while the vehicle is in motion,. These instruments are more effective and efficient in the long term than non-automatic weighing instruments. The development of automatic instruments emphasizes the need to confirm their metrological quality by calibrating and determining their measurement uncertainty. Calibration of such instruments also is carried on demand of different companies due to environment protection procedures (continuous totalizing automatic weighing instruments - belt weighers - used to control $\mathrm{CO}_{2}$ ).

\section{Calibration method}

In order to realize a calibration, the influence of various factors on the measured value should be taken into account. Such factors include the rate of operation of the instrument, type of material and nominal value of the testload.

The object of the calibration is the indication provided by the instrument in response to an applied load. The results are expressed in units of mass. The uncertainty of measurement depends significantly on properties of the calibrated instrument itself, the control instrument, the characteristics of the test loads, not only on the equipment of the calibrating laboratory.

In agreement with the client, the calibrating laboratory determines anticipated value of the uncertainty of measurement, which is appropriate in view of the use of the instrument and in view of the cost of the calibration.

Any such procedure must include, for a limited number of test loads, the determination of the error of indication and of the uncertainty of measurement assigned to these errors. The calibration should be processed as closely as possible to the weighing operations that are routinely being performed by the user.

The procedure may further include rules how to derive from the results advice to the user of the instrument with regard to the errors, and assigned uncertainty of measurement, of indications which may occur under normal conditions of use of the instrument.

Calibration of the complete WIM instrument is performed in the conditions of its use in a location and at the rate of operation requested by the client.

${ }^{*}$ Corresponding author: rafal.sarnecki@gum.gov.pl 
Before the application of the test procedure, extreme caution and safety in the dock entrance road and the weighing area should be guaranteed and provided.

When starting to calibrate, a test load should be applied to the instrument under specified conditions. Also, the total weight and axle load of the test vehicles constituting test loads should be determined, and the indication errors of WIM instrument should be calculated. The last step is to estimate the measurement uncertainty.

The weighing range is given by the values Max and Min. For Axle load, the Min=1000 $[\mathrm{kg}]$ and $\mathrm{Max}=20000[\mathrm{~kg}]$. In case of the mass od vehicle, Min=3500 and

Max $\leq 48000$ or as specified by national legislation.

Calibration of the complete WIM instrument is performed in the conditions of its use in a location agreed with the client. Before the application of the test procedure, extreme caution and safety in the dock entrance road and the weighing area should be guaranteed and provided.

\subsection{Determining the axle load $A x_{\text {ref }}$ of control vehicle}

Terminology and equation used in this article are based on:

- OIML R 76-1 [4]

- OIML R 134[1]

- EURAMET Calibration Guide No. 18 [2]

- OIML R 111-1[3]

The mass $A x_{\text {refi }}$ determined two axle vehicle Perform at least 10 load static measurements of $A x_{i}$ for the setting direction of the vehicle. The loads shall be determined in practice, at least two different loads: vehicle unloaded and the most loaded with standard weights or ballast.

Calculates the average value $A x_{i}$ for each axis of a 2 -axle vehicle representing the value $A x_{\text {ref.: }}$.

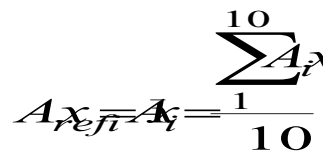

where: $A x_{i s}$ is value of the mass of the control vehicle axle load during the biaxial static weighing. Calculation of the total mass of the vehicle is realized by $V M_{i}$ :

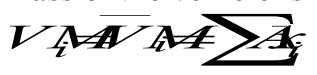

\subsection{Method of calibration the WIM instrument of the dynamic loading}

A complete WIM instrument is checked using control vehicle and other measuring equipment at rated operating conditions of WIM instrument, according to its characteristics. The mass of control vehicle should be such that it can be as close as possible to the maximum measuring range of the WIM instrument. The mass of control vehicle may be less adapted to customer needs. This should be agreed before starting the measuring procedure. Vehicles carrying liquids or other materials, which means harder-bones moves during the weighing may be used as a control vehicle only in duly substantiated cases (eg. customer needing, special application of WIM instrument). Speed of vehicles should be constant, without acceleration or braking, and should include the full speed range, including the minimum speed $V_{\min }$, and the typical speed on that road. If necessary (eg. according to the customer needs), the speed can be different (higher or lower than $V_{\max }$ ), but only with the acceptance of the customer and printing such information on the calibration certificate. For each of vehicle control, and for each set of the weight and speed and specified driving directions, takes at least $n=5$ passages:

- 3 runs down over the center of the load receptor,

- 1 ride left side of the load receptor,

- 1 ride right side of the load receptor. 


\section{MEASUREMENT RESULTS}

\subsection{Test of WIM instrument with 2-axles control vehicle dynamic loading}

Determines the error of axle $E_{A x i}$ and vehicle total mass $E_{V M i}$ from every passage of the individual vehicle and:

$$
\begin{aligned}
& E_{A x i}=A x_{i}-A x_{r e f i} \\
& E_{V M i}=V M_{i}-V M_{r e f}
\end{aligned}
$$

\section{UNCERTAINTY BUDGET}

\subsection{Standard uncertainty of discrete values}

The error is equal to difference between the value indicated by the instrumentin the certain entity and the reference load[2]:

$$
E=I-m_{\text {ref }}
$$

The equation of uncertainty has the form:

$$
u^{2}(E)=u^{2}(I)+u^{2}\left(m_{r e f}\right)
$$

In case of automatic weighing instruments, reference loads replaced by a value of control instrument and the formula for E calculation is:

$$
E=I-R_{K}
$$

where $R_{\mathrm{K}}$ is value from the control instrument and

$$
R_{K}=I_{K}-E_{K}
$$

where $E_{\mathrm{K}}$ is the error of the control instrument which means

Then the equation of uncertainty is:

$$
E=I-\left(I_{K}-E_{K}\right)
$$

where $u(I)$ is the uncertainty of the indication and

$$
u^{2}(E)=u^{2}(I)+u^{2}\left(R_{K}\right)
$$

$u\left(R_{K}\right)$ is the uncertainty of the value from the control instrument

\subsection{Standard uncertainty of indication}

$$
I=I_{L}+\delta I_{\operatorname{dig} L}+\delta I_{\text {rep }}-I_{0}-\delta I_{\text {dig } 0}
$$

All these correction have expected value of zero. Their standard uncertainties are: $\delta I_{\text {digo } 0}$ stands for the rounding error at zero loading

$$
u\left(d_{\text {dig }}\right)=\frac{d}{2 \sqrt{3}}
$$

or

$$
u\left(\Phi_{\text {dig }}\right)=\frac{d_{T}}{2 \sqrt{3}}
$$

$\delta I_{\text {digL }}$ stands for the rounding error of indication at a load

$$
u\left(\Phi_{\text {dig }}\right)=\frac{d_{1}}{2 \sqrt{3}}
$$

or

$$
u\left(\delta I_{\operatorname{digL}}\right)=\frac{d_{T}}{2 \sqrt{3}}
$$

$\delta I_{\text {rep stands for the error of repeatability }}$

$$
u\left(\delta I_{\text {rep }}\right)=\mathrm{s}\left(I_{j}\right)
$$


Standard deviation calculation is carried out form a series of calculated errors for each entity (gross mass or axle load).

\subsection{Standard uncertainty of a value from the control instrument $u\left(R_{\mathrm{K}}\right)$}

$$
u\left(R_{K}\right)^{2}=u_{\text {opak }}^{2}+u_{d 0}^{2}+u_{d I}^{2}+u_{E I}^{2}
$$

where $u\left(R_{K}\right)$ is equal to the uncertainty of the instrument in use. The uncertainty of the control instrument value $u_{E I}$ for the given load (the value is obtained from the control instrument calibration certificate) is expanded by an uncertainty of indication.

The value of the uncertainty taken from a calibration certificate is valid only for a control instrument intended for weighing the gross mass of a reference vehicle. follows.

The uncertainty of a control instrument for weighing axle load shall be obtained as

$$
\%=\frac{10390 \%}{\sqrt{3}}
$$

where: mpe is the maximum permissible error for the given load in service;

0,005 is the value resulting from external effects;

$I$ is the indication.

The individual results of measurement are considered to be as correlated quantities (coefficient: $r=1$ ) and summing the uncertainties of individual axles shall be as follows:

The expanded uncertainty:

$$
u_{C}=u_{1}+u_{2}+\ldots+u_{\mathrm{n}}
$$

$$
U=2 \times u_{C}
$$

\subsection{Combined standard uncertainty}

$$
u\left(E_{I}\right)^{2}=u_{\text {opak }}^{2}+u_{d 0}^{2}+u_{d I}^{2}+\left(u_{\text {opak }}^{2}+u_{d 0}^{2}+u_{d I}^{2}+u_{E I}^{2}+u\left(m_{T}\right)^{2}\right)
$$

where items in brackets represent an uncertainty of the control instrument.

\section{Implementation of HS- WIM}

In last year Central Office of Measures intensive effort to establish legal metrology control of high speed weighing in motion. Roads are being damaged by overweight trucks. Too heavy trucks also are dangerous because of longer way of reducing their speed. To reduce wear of road surface and increase safety, Poland decided to focus effort on implementing high-speed weighing of road vehicles subject to legal metrology. To achieve this, it is necessary to implement devices enabling the imposition of penalties on road users destroying the surface by moving overloaded vehicles, for legal metrological control. The implementation of such systems will improve both road safety and indirectly limit the number of road users moving overloaded vehicles. In Poland currently over hundred HS-WIM instruments work as "preselection", which means they measure load and Road and Traffic Security Guard must repeat measurment on legally approved non-automatic weighing instruments (NAWIs). Drivers know when road inspectors are in work and too heavy trucks avoid control waiting for officers to end their work so the best solution is automatic $24 \mathrm{~h}$ /day system which controls loads the same way like it is speed measuring.

\subsection{The method of carrying out the measurement}

There were vehicles: an empty bus, a bus with a $500 \mathrm{~kg}$ standard, a truck with a weight of $3000 \mathrm{~kg}$ and a weight of $39,000 \mathrm{~kg}$. The vehicles passed through two measuring stations, turned back and passed them again. The instruments installed on the gantry identified the vehicle and measured its length. The vehicle was passing through transducers installed in the roadway. 
The measurement was repeated 10 times. The time of the vehicle's passage through the transducers was also monitored. The results were recorded in the system that was available for the Inspectorate of Road Transport.

Fig1. and Fig2. Show error points for each measurement. Testing different types of load sensors shows that is possible to build automatic load measurment system. Accuracy of systems which are now used is $3-5 \%$ while overweight of trucks vary between 10 and $20 \%$, so for road inspection it is efficient accuracy.

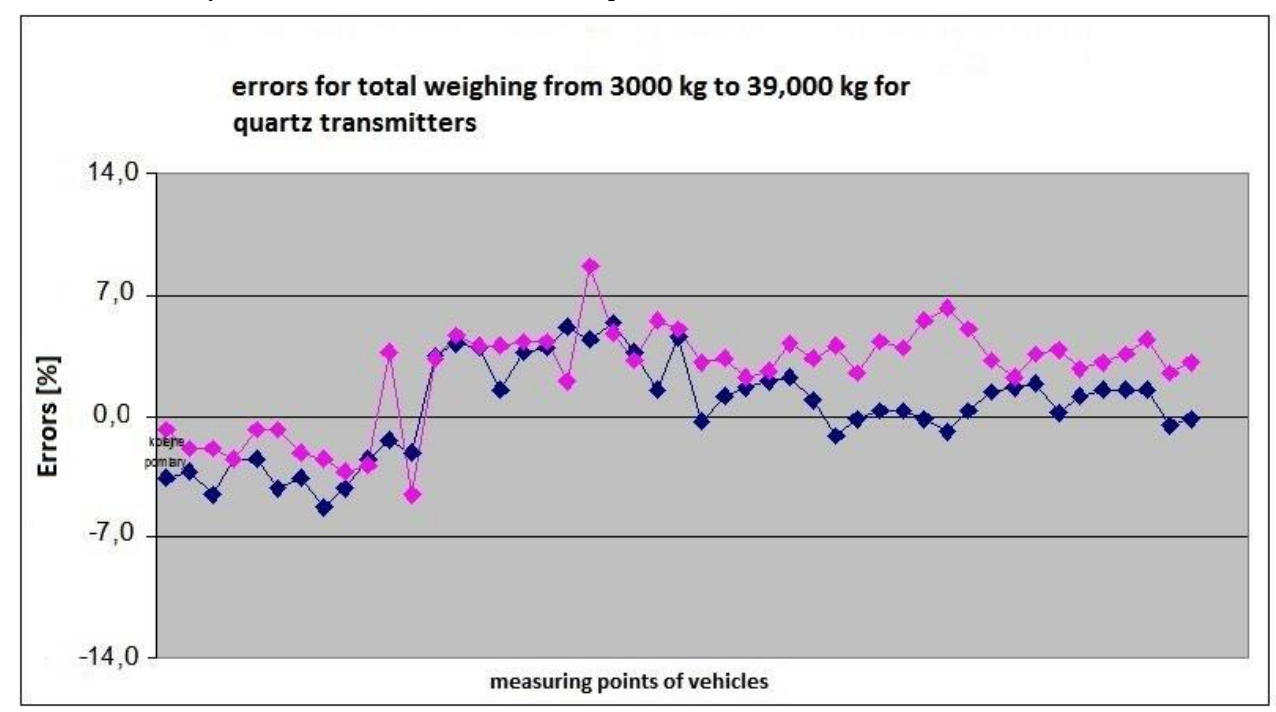

Fig1. Errors for total weight for quart transmitters.

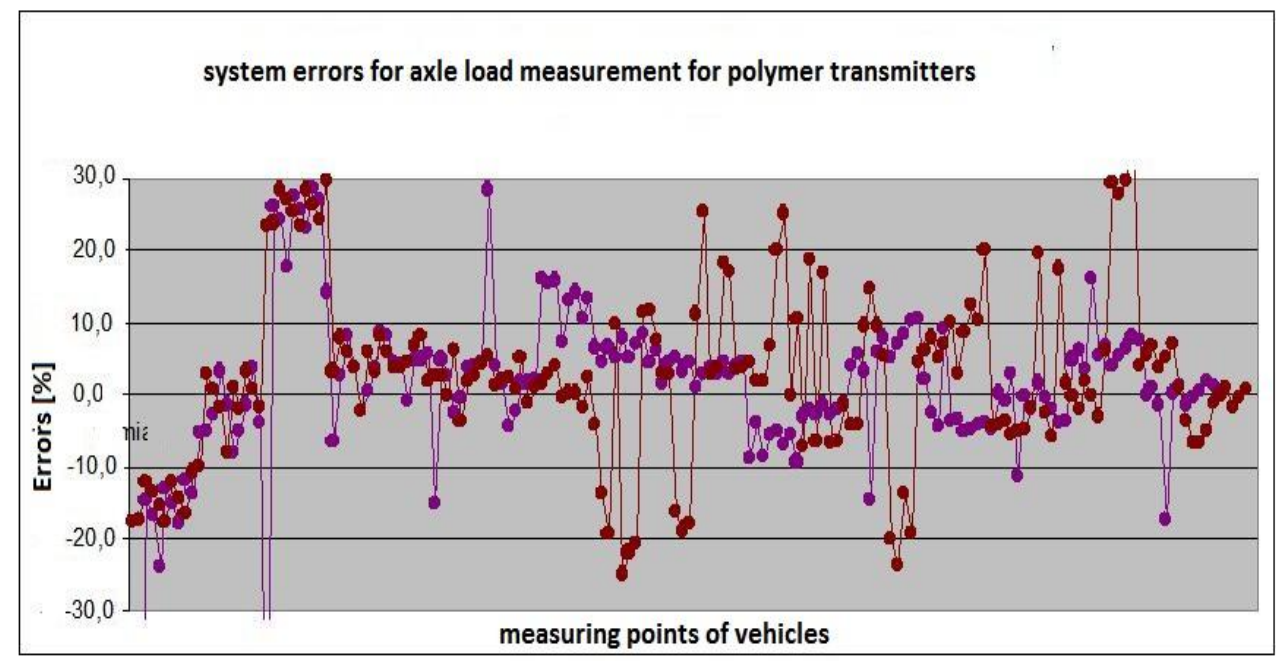

Fig2. Errors for total weight for polymer transmitters.

\subsection{Plans for testing laboratory of HS-WIM}

Central Office of Measures plans to build a complex that allows testing all devices in this type of instruments.

The complex will include:

- test road

- laboratory of speed measurement 
- warehouses

- laboratory of testing electrical interference

- laboratory of total mass measurement

Full complex is shown on Fig3.
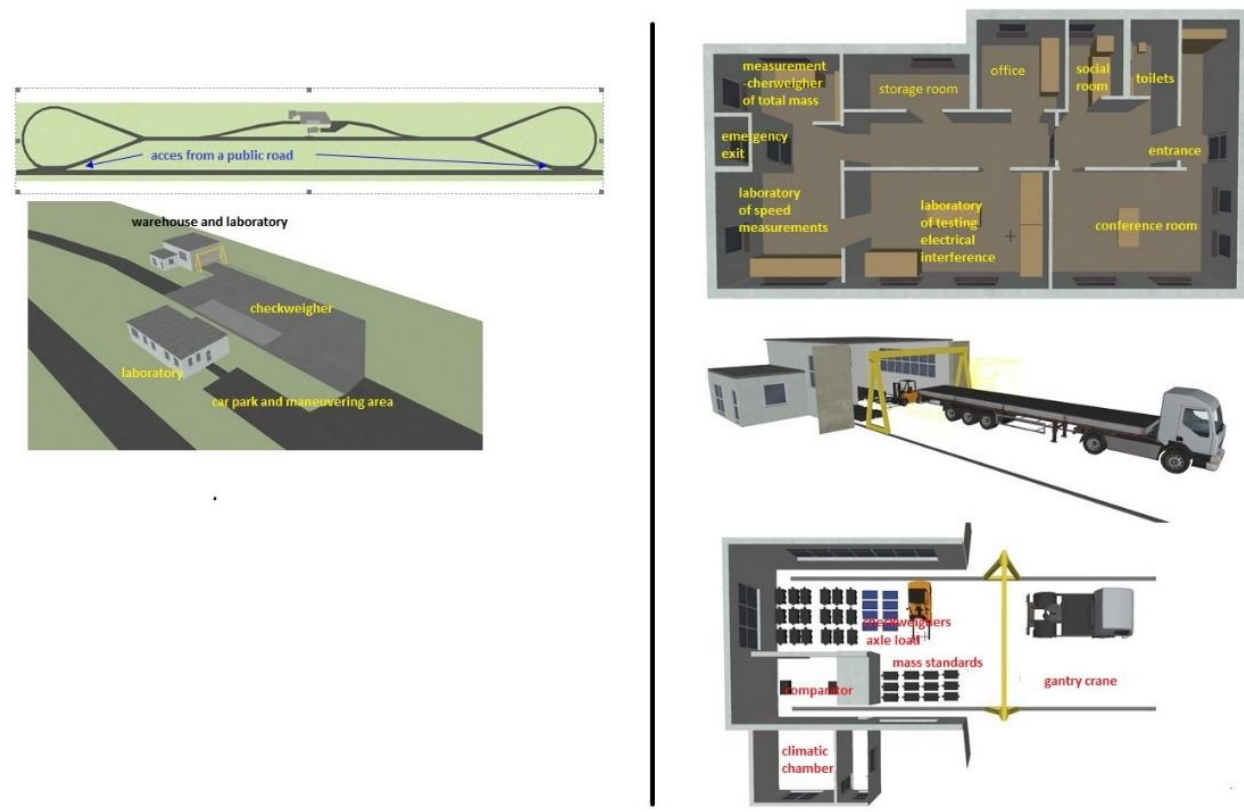

Fig3. Laboratory complex for testing HS-WIM.

\section{Conclusion}

The method of calibration of automatic instruments described in the article will improve weighing process control in different industries such as production pre-packed products and transport. The calibration laboratories be accredited by accreditation bodies based on the harmonised guidance. In the case of AWI, the uncertainty of measurement depends to a large extent on the properties of the calibrated instrument itself, the characteristics of test loads, the control instrument, and not only the other possibilities of the calibration laboratory.

Several methods for determination of the reference mass are available however, it is necessary to look for a suitable compromise between the accuracy and simplicity of the selected method. Additionally, a density of the test load does not need to be known without significant influence to the measurement uncertainty. Significant progress has been made in creating the guide for automatic balances. Mass Laboratory in Central Office of Measures wants to convince state to build laboratory which is necessary to carry further tests and carry type approval in future. Building such testing laboratory will cost a lot of money but implementing working HS-WIM system should prevent much bigger cost of damaging roads.

\section{References}

1. OIML R 134-1, Automatic instruments for weighing road vehicles in motion and measuring axle loads. Part 1: Metrological and Technical Requirements - Tests (2006)

2. EURAMET Calibration Guide No. 18, Guidelines on the Calibration of Non-Automatic Weighing Instruments, Version 4.0 (1/205)

3. OIML R 111-1, Weights of classes $E_{1}, E_{2}, F_{1}, F_{2}, M_{1}, M_{1-2}, M_{2}, M_{2-3}$ and $M_{3}$. Part 1: Metrological and Technical Requirements, (2004) 
MATEC Web of Conferences 182, 02005 (2018)

https://doi.org/10.1051/matecconf/201818202005

$17^{\text {th }}$ International Conference Diagnostics of Machines and Vehicles

4. OIML R 76-1, Non-automatic weighing instruments. Part 1: Metrological and Technical Requirements - Tests 206) 